\title{
Extending electro-optic detection to ultrashort electron beams
}

\author{
M. H. Helle, ${ }^{*}$ D. F. Gordon, D. Kaganovich, and A. Ting \\ Plasma Physics Division, Naval Research Laboratory, Washington, D.C. 20375, USA \\ (Received 29 September 2011; revised manuscript received 23 February 2012; published 3 May 2012)

\begin{abstract}
We propose a technique to extend noninvasive electro-optic detection of relativistic electron beams to bunch lengths of $\simeq 10 \mathrm{fs}$. This is made possible by detecting the frequency mixing that occurs between the optical probe and the space charge fields of the beam, while simultaneously time resolving the resulting mixed frequency signal. The necessary formalism to describe this technique is developed and numerical solutions for various possible experimental conditions are made. These solutions are then compared to simulation results for consistency. Finally, the method to reconstruct the original bunch profile from the proposed diagnostic is discussed and an example showing a 15 fs test beam reconstructed to within an accuracy of $15 \%$ is given.
\end{abstract}

DOI: 10.1103/PhysRevSTAB.15.052801

\section{INTRODUCTION}

There has been considerable work over the past decade on the development of a single shot, noninvasive, optical technique to measure the longitudinal profile of an electron bunch. This has proved to be a difficult task as bunch lengths have dropped below $100 \mathrm{fs}$ and in the case of laser wakefield accelerators are sub-10 fs [1]. One technique that has drawn significant attention has been electro-optic sampling (EOS). EOS is a common technique used to measure short pulse terahertz $(\mathrm{THz})$ fields. Recently, this has been adapted to the longitudinal profiling of accelerated charged particle beams. EOS has been used to characterize beams either directly, by measuring their self-fields [2-11], or indirectly by measuring the $\mathrm{THz}$ fields generated from transition radiation diagnostics $[12,13]$. In the case of the former, the particle beam's longitudinal profile can be deduced from its self-fields due to the fact that for sufficiently relativistic beams the temporal structure of the selffields matches the temporal structure of the beam [14]. The technique is commonly described as being analogous to an electro-optic modulator based on Pockels effect [15]. The electric field of interest induces a time-varying birefringence within an electro-optic crystal that is being probed optically. The field's profile is then extracted from the optical signal using ellipsometry methods, a few of which will be discussed in the next section.

\section{A. Current techniques}

Schematics for three of the most widely used detection schemes are contained in Berden et al. [7]. All these

\footnotetext{
*Corresponding author. mike.helle@nrl.navy.mil
}

Published by the American Physical Society under the terms of the Creative Commons Attribution 3.0 License. Further distribution of this work must maintain attribution to the author(s) and the published article's title, journal citation, and DOI.
PACS numbers: 41.75.Ht, 29.40.Wk, 29.40.Gx, 42.65.Re

schemes rely on polarization changes due to the Pockels effect to extract the bunch profile. Frequency mixing, if considered at all, is regarded as a parasitic and undesirable effect [16]. In the technique proposed here, frequency mixing is accounted for at every stage of the process and is considered beneficial for bunch profile extraction.

The earliest detection method was the balanced photodiode detection scheme $[2,15,17]$. When the charged particle beam is present, a birefringence is induced within the crystal. An ultrashort optical probe sees this induced birefringence, which shows up as a change in the splitting ratio recorded by the balanced photodiode. By changing the delay of the probe pulse, the beam profile can be mapped out. The disadvantage of this technique is that it requires scanning of the probe and thus is not single shot. Additionally, it requires that the probe is much shorter than the bunch of interest. This technique can be adapted to single shot applications by using a long probe pulse and relying on the bandwidth of the balanced photodiode to resolve the bunch profile. However, most balanced photodiodes have bandwidths in the 100's of $\mathrm{MHz}$ and thus only bunches greater than a few nanoseconds would be appropriate.

The next advancement in EOS was the development of a single shot technique that relies on spectral decoding $[3,7,8,16]$. Here instead of an ultrashort pulse, a stretched pulse is used as a probe and only the polarization state corresponding to the $1 \overline{1} 0$ axis is examined. In this arrangement, the fields from the charged particle bunch act to modulate the intensity profile of the stretched probe. Since the stretched probe is produced by placing a linear chirp on an ultrashort pulse, each frequency within the pulse corresponds to a point in time and thus the bunch profile is imprinted on the spectrum of the beam. By precisely knowing the chirp placed on the probe the bunch profile can be extracted directly from the spectrum; however, frequency mixing modifies the spectrum and reduces the accuracy of this technique. 
The latest refinement to EOS is temporal decoding [6-8]. As with the previous technique, a stretched pulse is used to probe the nonlinear crystal. The difference is that the profile is now extracted by cross correlating an ultrashort pulse with the probe pulse in a second harmonic crystal. Cross correlation at a wide angle has the effect of transferring the temporal structure of the probe into the spatial structure of the second harmonic pulse. The second harmonic is then recorded by a CCD and the bunch profile is extracted. By not relying on the spectral content, temporal decoding side steps the issue of frequency mixing, but fails to take advantage of it.

The development of these decoding methods has lead to experimentally measured electron bunches as short as 120 and $60 \mathrm{fs}$ using the electro-optic crystals ZnTe and GaP, respectively $[7,10,11]$. Unfortunately, this represents what is the experimentally verified lower bound for these decoding methods. A discussion of the reasons for this limitation as well as nonlinear processes that will allow for the measurement of even shorter bunches follows.

\section{B. Limitations}

For a material to exhibit the Pockels effect it must lack inversion symmetry. Ionic crystals are therefore an obvious choice and tend to have relatively high electro-optic coefficients. However, these same structural properties lead to the existence of a transverse optical phonon frequency in the $\mathrm{THz}$ frequency range. The phonon frequency shows up as a resonance in the dispersion relation [18-20]. This is clearly seen in Figs. 1(a) and 1(b) for the refractive index and electro-optic coefficient of ZnTe in the $\mathrm{THz}$ range, respectively.

The resonance effect is immaterial when trying to measure electron bunches $\gtrsim 120$ fs for ZnTe and $\gtrsim 60$ fs for $\mathrm{GaP}$. This is due to the fact that, in the frequency domain, the corresponding self-fields' frequency components are contained mostly to the region below the resonance. Thus, for any given electro-optical material, a bunch length longer than $\frac{1}{\nu_{\mathrm{TO}}}$ can easily be extracted. Here $\nu_{\mathrm{TO}}$ is the transverse optical phonon frequency. However, for shorter beams this is no longer the case. For these types of beams the resonance has the effect of distorting the temporal profile of the selffields as they propagate through the crystal. Additionally, certain frequencies can be suppressed by the slippage between the phase/group velocities of the self-fields and the optical probe. These effects are often discussed in terms of the so-called electro-optic response function, $G(\omega)$, that approximates the ratio of the reconstructed spectrum to the actual spectrum [15,17]. An example of such a response function plotted for various thicknesses of $\mathrm{ZnTe}$ is given in Brunken et al. [17]. For various electro-optic materials, the response function becomes more favorable for thinner crystals. It has been the focus of most ongoing research to obtain thinner and thinner crystals to measure shorter bunches. This leads to reduced signal and does not resolve the ultimate issue, namely the resonance $[12,15]$.

Fortunately, the picture described above is not complete in its treatment of the mixing process that occurs between the self-fields and the optical probe. The Pockels effect is actually a low frequency limiting case of three-wave mixing, and for the frequency components being considered here it is no longer valid [22]. A more thorough consideration of the interaction must include both sum and difference frequency generation [23]. The following sections present a picture of the interaction using the formalism of three-wave mixing. From this picture a new technique is proposed that uses frequency mixing to extend beyond the resonance frequency and is capable of measuring beams that are $<\frac{1}{\nu_{\mathrm{TO}}}[24]$.

\section{FREQUENCY MIXING}

EOS relies on the fact that the particle beam is sufficiently relativistic that its self-fields' profile matches its longitudinal profile. In addition to the enhanced transverse electric field, in the laboratory frame, a magnetic field component is introduced. At these relativistic velocities,

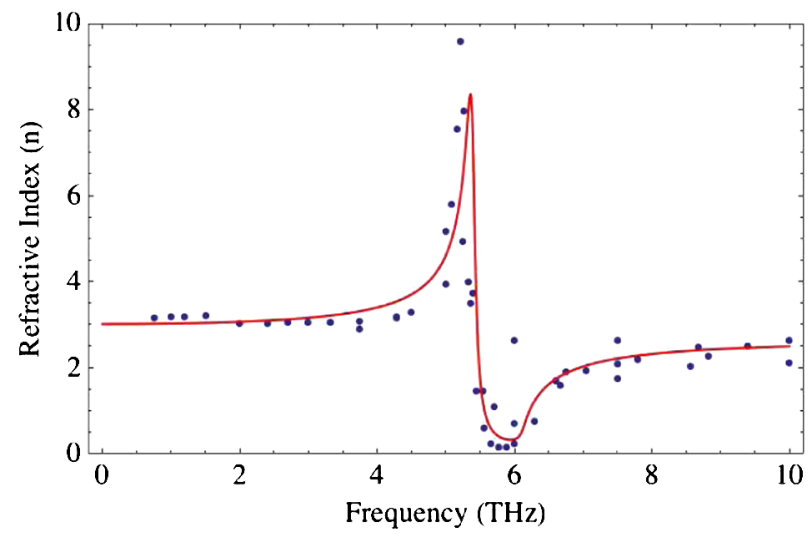

(a)

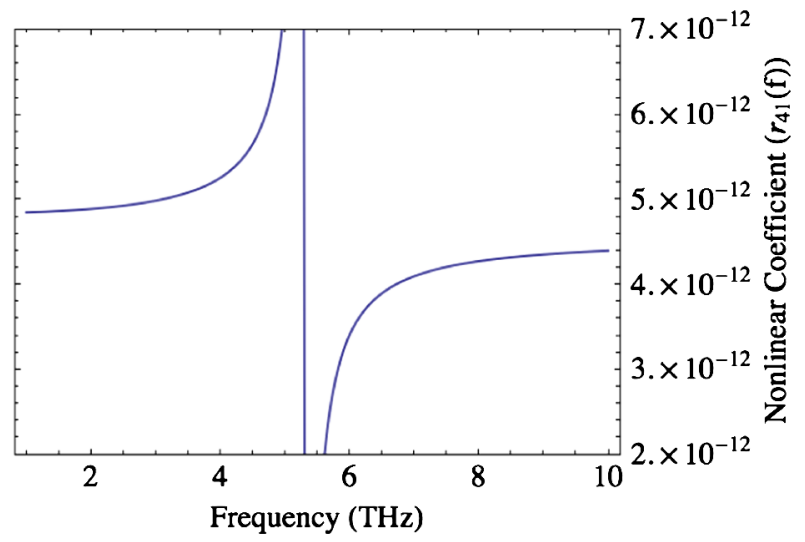

(b)

FIG. 1. Plots showing the refractive index (a) and electro-optic coefficient $r_{41}$ (b) of ZnTe at various frequencies [17,44]. 
the combined transverse electric and magnetic self-fields become indistinguishable from an infinite radially polarized electromagnetic pulse [14]. Using this fact, EOS of longitudinal profiles will be described not as a Pockels effect, but more generally as the nonlinear mixing of an electromagnetic pulse with an optical probe. The result is the generation of fields at the sum and difference frequency as discussed in [22,25-28].

Let us begin by looking at the nonlinear mixing of the self-fields with a chirped laser probe within a noncentrosymmetric, second order material. Here the depletion of the self-fields and the probe fields is neglected. As a consequence of this, the probe propagates through the material according to its linear wave equations. Also, a slowly varying envelope approximation, where the time scale on which the envelope evolves is long compared to the optical cycle, is used. The coordinate system is set up such that the fields are traveling along the $z$ axis and the interface between vacuum and the nonlinear material is located at $z=0$. The fields are then defined as follows:

$$
\begin{aligned}
& \text { Particle beam self-fields: } \mathbf{E}_{1}(z, t)=\int_{-\infty}^{\infty} A_{1}\left(\omega_{1}\right) e^{l k\left(\omega_{1}\right) z} e^{-l \omega_{1} t} d \omega_{1} \hat{\mathbf{p}}_{1} \\
& A_{1}\left(\omega_{1}\right)=\frac{2}{1+n\left(\omega_{1}\right)+\imath \kappa\left(\omega_{1}\right)} \int_{-\infty}^{\infty} E_{1}(0, t) e^{\imath \omega_{1} t} d t \\
& \text { Optical probe: } \mathbf{E}_{2}(z, t)=\int_{-\infty}^{\infty} A_{2}\left(\omega_{2}-\omega_{0}\right) e^{\imath k\left(\omega_{2}\right) z} e^{-\imath\left(\omega_{2}-\omega_{0}\right) t} d \omega_{2} \hat{\mathbf{p}}_{2} \\
& A_{2}\left(\omega_{2}-\omega_{0}\right)=\frac{2}{1+n\left(\omega_{2}\right)+\imath \kappa\left(\omega_{2}\right)} \int_{-\infty}^{\infty} E_{2}(0, t) e^{l\left(\omega_{2}-\omega_{0}\right) t} d t \\
& \text { Sum frequency: } \mathbf{E}_{3}(z, t)=\int_{-\infty}^{\infty} A_{3}\left(\omega_{3}-\omega_{0}\right) e^{\imath k\left(\omega_{3}\right) z} e^{-l\left(\omega_{3}-\omega_{0}\right) t} d \omega_{3} \hat{\mathbf{p}}_{\mathrm{NL}} \\
& \text { Difference frequency: } \mathbf{E}_{4}(z, t)=\int_{-\infty}^{\infty} A_{4}\left(\omega_{4}-\omega_{0}\right) e^{\imath k\left(\omega_{4}\right) z} e^{-l\left(\omega_{4}-\omega_{0}\right) t} d \omega_{4} \hat{\mathbf{p}}_{\mathrm{NL}} \text {, } \\
& \text { Sum frequency: } A_{3}\left(\ell, \omega_{3}\right)=\imath \frac{\omega_{3}^{2}}{c^{2} k_{3}^{\prime}\left(\omega_{3}\right)} \chi_{i j k}^{(2)}\left(\omega_{3} ; \omega_{2}, \omega_{1}\right) \frac{e^{\imath \Delta k_{+} \ell}-1}{\imath \Delta k_{+}} e^{-k^{\prime \prime}\left(\omega_{3}\right) \ell} A_{1}\left(\omega_{1}\right) A_{2}\left(\omega_{2}-\omega_{0}\right) \\
& \text { Difference frequency: } A_{4}\left(\ell, \omega_{4}\right)=l \frac{\omega_{4}^{2}}{c^{2} k_{4}^{\prime}\left(\omega_{4}\right)} \chi_{i j k}^{(2)}\left(\omega_{4} ; \omega_{2},-\omega_{1}\right) \frac{e^{\imath \Delta k_{-} \ell}-1}{\imath \Delta k_{-}} e^{-k^{\prime \prime}\left(\omega_{4}\right) \ell} A_{1}^{*}\left(\omega_{1}\right) A_{2}\left(\omega_{2}-\omega_{0}\right) \text {, }
\end{aligned}
$$

where $k(\omega)=k^{\prime}(\omega)+\imath k^{\prime \prime}(\omega), \Delta k_{+}=k_{1}\left(\omega_{1}\right)+k_{2}\left(\omega_{2}\right)-k_{3}\left(\omega_{2}+\omega_{1}\right), \Delta k_{-}=-k_{1}\left(\omega_{1}\right)+k_{2}\left(\omega_{2}\right)-k_{3}\left(\omega_{2}-\omega_{1}\right)$, and $\chi_{i j k}^{(2)}$ is the second order nonlinear susceptibility. The above equations are taken from Ref. [29] and take into account the effect of absorption on the nonlinear mixing process.

By making the substitution,

$$
\text { Sum frequency: } \omega_{1}=\Omega \text { and } \omega_{2}=\omega-\Omega \quad \text { Difference frequency: } \omega_{1}=\Omega \text { and } \omega_{2}=\omega+\Omega \text {, }
$$

and following the derivation outlined in Ref. [22], the total field generated from nonlinear wave mixing is

$$
\begin{aligned}
\mathbf{E}_{\text {mix }}(\ell, t)=\mathbf{E}_{3}(\ell, t)+\mathbf{E}_{4}(\ell, t)= & \int_{-\infty}^{\infty} \int_{-\infty}^{\infty} \frac{\omega^{2}}{c^{2}\left|k^{\prime}(\omega)\right|} e^{l k(\omega) \ell} \operatorname{Re}\left[\chi^{(2)}(\omega ; \Omega, \omega-\Omega) \cdot \hat{\mathbf{p}}_{1} \cdot \hat{\mathbf{p}}_{2}\right] \\
& \times \frac{e^{\imath \Delta k_{+}(\Omega, \omega) \ell}-1}{\imath \Delta k_{+}(\Omega, \omega)} A_{1}(\Omega) e^{-\imath \Omega(t+\tau)} A_{2}\left(\omega-\Omega-\omega_{0}\right) e^{-l\left(\omega-\Omega-\omega_{0}\right) t} d \Omega d \omega,
\end{aligned}
$$

where $\tau$ is the separation in time between the self-fields and the probe. 
The above expression can be solved numerically; however, by first making a couple of approximations the solution can become less intensive. Let us begin by assuming that dispersion is small over the optical frequencies of interest and that $\omega \approx \omega_{0}$. The latter is consistent with the slowly varying envelope approximation made above and with the relatively narrow bandwidth probe pulses used experimentally $[22,29,30]$. From these assumptions the following approximations can be made:

$$
\begin{aligned}
k(\omega) & \approx k\left(\omega_{0}\right)+\left(\frac{d k}{d \omega}\right)_{\omega_{0}}\left(\omega-\omega_{0}\right) \\
& =k\left(\omega_{0}\right)+\frac{n_{g}\left(\omega_{0}\right)}{c}\left(\omega-\omega_{0}\right),
\end{aligned}
$$

$$
\begin{aligned}
\Delta k_{+} & =-k(\omega)+k(\Omega)+k(\omega-\Omega) \\
& \approx k(\Omega)-\frac{n_{g}\left(\omega_{0}\right)}{c} \Omega
\end{aligned}
$$

and

$$
\operatorname{Re}\left[\chi_{i j k}^{(2)}(\omega ; \Omega, \omega-\Omega)\right] \approx \operatorname{Re}\left[\chi_{i j k}^{(2)}\left(\omega_{0} ; \Omega, \omega_{0}-\Omega\right)\right]
$$

where $n_{g}$ is the group index of the material. Applying these approximations and accounting for both positive and negative values of $\omega_{0}$, Eq. (7) becomes

$$
\begin{aligned}
E_{\text {mix }}(t, \ell) \approx & l \int_{-\infty}^{\infty} \frac{\omega_{0}^{2}}{c^{2}\left|k^{\prime}\left(\omega_{0}\right)\right|} \operatorname{Re}\left[\chi_{i j k}^{(2)}\left(\omega_{0} ; \Omega, \omega_{0}-\Omega\right)\right] \frac{e^{l\left[k(\Omega)-n_{g}\left(\omega_{0}\right) \Omega / c\right] \ell}-1}{l\left[k(\Omega)-n_{g}\left(\omega_{0}\right) \Omega / c\right]} A_{1}(\Omega) e^{-\imath \Omega(t+\tau)} \\
& \times \int_{-\infty}^{\infty}\left[A_{2}\left(\omega-\Omega-\omega_{0}\right) e^{\imath k\left(\omega_{0}\right) \ell} e^{l n_{g}\left(\omega_{0}\right) \ell / c\left(\omega-\omega_{0}\right)} e^{\imath \omega_{0} t}+A_{2}\left(\omega-\Omega+\omega_{0}\right) e^{\imath k\left(-\omega_{0}\right) \ell} e^{l n_{g}\left(\omega_{0}\right) \ell / c\left(\omega+\omega_{0}\right)} e^{-l \omega_{0} t}\right] \\
& \times e^{-\imath\left(\omega-\Omega-\omega_{0}\right) t} d \omega d \Omega .
\end{aligned}
$$

The integral over $\omega$ can be solved analytically by assuming a Gaussian probe pulse with a chirp. Namely,

$$
E_{2}(0, t)=E_{0} e^{-t^{2} / 2 \sigma^{2}} e^{\imath \beta t^{2}} e^{l \omega_{0} t},
$$

where $\sigma^{2}$ is the variance and $\beta$ is the linear chirp parameter [31].

For such a probe pulse, the integral becomes

$$
\begin{aligned}
& \int_{-\infty}^{\infty}\left[A_{2}\left(\omega-\Omega-\omega_{0}\right) e^{l k\left(\omega_{0}\right) \ell} e^{l n_{g}\left(\omega_{0}\right) \ell / c\left(\omega-\omega_{0}\right)} e^{l \omega_{0} t}+A_{2}^{*}\left(\omega-\Omega-\omega_{0}\right) e^{l k\left(-\omega_{0}\right) \ell} e^{l n_{g}\left(\omega_{0}\right) \ell / c\left(\omega+\omega_{0}\right)} e^{-l \omega_{0} t}\right] e^{-l(\omega-\Omega) t} d \omega \\
& =E_{0} e^{-\left[t-n_{g}\left(\omega_{0}\right) \ell / c\right]^{2} / 2 \sigma^{2}} e^{l n_{g}\left(\omega_{0}\right) \Omega \ell / c}\left(e^{l\left[t-n_{g}\left(\omega_{0}\right) \ell / c\right]^{2} \beta} e^{l k\left(\omega_{0}\right) \ell} e^{l \omega\left[t-n_{g}\left(\omega_{0}\right) \ell\right] / c}+e^{-l\left[t-n_{g}\left(\omega_{0}\right) \ell / c\right]^{2} \beta} e^{-l k\left(\omega_{0}\right) \ell} e^{-l \omega\left[t-n_{g}\left(\omega_{0}\right) \ell / c\right]}\right) .
\end{aligned}
$$

Here, $A_{2}\left(\omega-\Omega+\omega_{0}\right)=A_{2}^{*}\left(\omega-\Omega-\omega_{2}\right)$ is used. Plugging this into Eq. (10) yields

$$
\begin{aligned}
E_{\text {mix }}\left(t^{\prime}-\tau, \ell\right) \approx & l \frac{\omega_{0}^{2}}{c^{2}\left|k^{\prime}\left(\omega_{0}\right)\right|} E_{0} e^{-\left[t^{\prime}-\tau-n_{g}\left(\omega_{0}\right) \ell / c\right]^{2} / 2 \sigma^{2}}\left(e^{l\left[t^{\prime}-\tau-n_{g}\left(\omega_{0}\right) \ell / c\right]^{2} \beta} e^{l k\left(\omega_{0}\right) \ell} e^{l \omega\left[t^{\prime}-\tau-n_{g}\left(\omega_{0}\right) \ell / c\right]}\right. \\
& \left.+e^{-l\left[t^{\prime}-\tau-n_{g}\left(\omega_{0}\right) \ell / c\right]^{2} \beta} e^{-l k\left(\omega_{0}\right) \ell} e^{-l \omega\left[t^{\prime}-\tau-n_{g}\left(\omega_{0}\right) \ell / c\right]}\right) \\
& \times \int_{-\infty}^{\infty} \operatorname{Re}\left[\chi_{i j k}^{(2)}\left(\omega_{0} ; \Omega, \omega_{0}-\Omega\right)\right] e^{l n_{g}\left(\omega_{0}\right) \Omega \ell / c} \frac{e^{\imath\left[k(\Omega)-n_{g}\left(\omega_{0}\right) \Omega / c\right] \ell}-1}{l\left[k(\Omega)-n_{g}\left(\omega_{0}\right) \Omega / c\right]} A_{1}(\Omega) e^{-l \Omega t^{\prime}} d \Omega,
\end{aligned}
$$

where the substitution $t^{\prime}=t+\tau$ has been made. The above general solution can be applied to any noncentrosymmetric nonlinear material with arbitrary orientation.

\section{BANDWIDTH MIXING CROSS-CORRELATION FREQUENCY RESOLVED OPTICAL GATING (BMX-FROG)}

In this section, the proposed experimental apparatus shown in Fig. 2 will be considered. This setup was first introduced in [24]. A chirped optical pulse is split into two parts. The first part is compressed into an ultrashort pulse for use as an optical gate. The second part passes through a set of cross polarizers sandwiching a nonlinear crystal. For the case presented here, zinc telluride ( $\mathrm{ZnTe}$ ) will be used, but any other crystal of the $\overline{4} 3 \mathrm{~m}$ crystal class could be easily substituted. ZnTe was chosen due to its wide availability and its lower TO frequency when compared to GaP. The lower TO frequency aids in illustrating the above resonance mixing that occurs. The first polarizer is set up such that the optical probe's polarization is perpendicular to the direction of particle beam's self-fields within the ZnTe. The ZnTe is cut such that its 110 axis is aligned to the direction of propagation and its $\overline{1} 10$ and 001 axes are aligned to the self-field's direction and the probe's polarization, respectively. This type of geometry has the effect of extinguishing the probe while allowing the mixed field to pass freely through the second polarizer. 


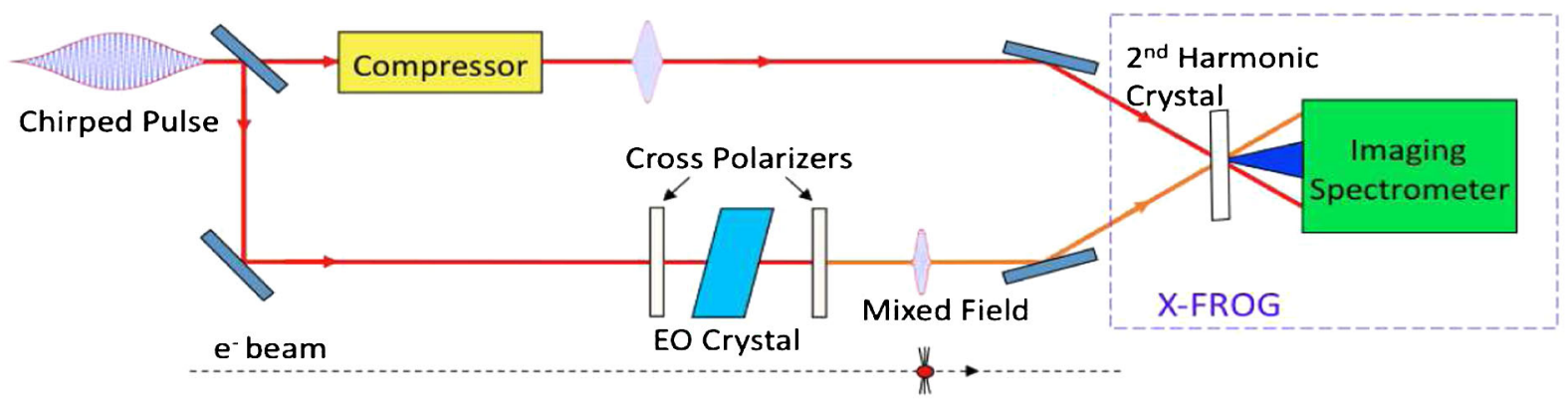

FIG. 2. BMX-FROG setup used to measure ultrashort charged particle bunches.

The signal is decoded by a cross-correlation frequency resolved optical gate (X-FROG). The use of a FROG to decode the EO signal has been proposed previously by Bolton et al. [32,33]. In these works a polarization-gated (PG) FROG was suggested. A PG FROG works by cross correlating the signal with itself in a third-order nonlinear material. The issue with using a PG-FROG is that it requires a significantly large signal $(\sim \mathrm{mJ})$ to generate an output. Such a requirement could lead to undesirable multiphoton processes and damage of the EO crystal. Also, the retrieval algorithm is difficult to implement and does not reliably converge for complex pulse profiles. An X-FROG is used here instead since it has a more robust retrieval algorithm and is compatible with small signals $(<\mathrm{nJ})$. These characteristics are due to the reliance on a lower order $\chi^{(2)}$ effect (2nd harmonic generation) instead of a $\chi^{(3)}$ effect (cross-phase modulation), as well as an external gate that can have its intensity increased to amplify the X-FROG output [34].

Since this setup combines the bandwidth mixing of the chirped probe and self-fields with an X-FROG, we refer to it as a bandwidth mixing cross-correlation frequency resolved optical gate (BMX-FROG). The BMX-FROG works by cross correlating the mixed field with the gate pulse within a second harmonic crystal. The second harmonic signal is then placed through an imaging spectrometer that produces an image of time versus frequency. From this image, both amplitude and phase information for each frequency component in the pulse is retrieved. By coupling the information provided by frequency mixing with amplitude and phase information, this technique is capable of reconstructing the profile for bunches below the lower bound set by the TO resonance that currently limits other EO detection techniques.

To model this, the reduced equation given in Eq. (12) was solved numerically. The code uses the dispersion and second order susceptibility relationships discussed in Refs. [35,36]. A comparison between the experimental data for the refractive index and extinction coefficient and the equations used for the numerical solution is shown in Fig. 3 [37]. The experimental data was manually extracted from tables in Ref. [38]. The equations themselves are reproduced below:

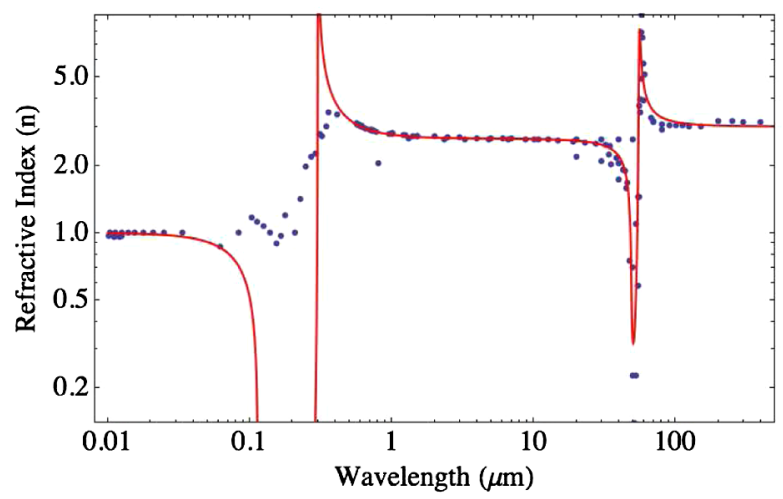

(a)

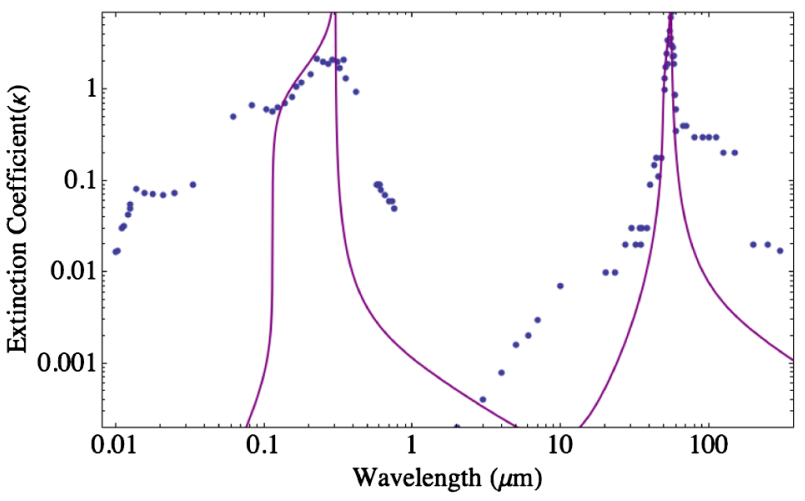

(b)

FIG. 3. Comparison between experiment data and fits for the refractive index (a) and extinction coefficient (b) for ZnTe. The experimental data was extracted from Ref. [38]. 


$$
\begin{aligned}
n(\omega)= & \operatorname{Re}[\sqrt{\epsilon(\omega)}] \quad \kappa(\omega)=\operatorname{Im}[\sqrt{\epsilon(\omega)}] \\
\epsilon(\omega)= & 1+\frac{\omega_{\mathrm{THz}}^{2}}{\Omega_{\mathrm{THz}}^{2}-\imath \gamma_{\mathrm{THz}} \omega / \omega_{\epsilon}^{2}-\omega^{2} / \omega_{\epsilon}^{2}} \\
& +\frac{\omega_{\mathrm{opt}}^{2}}{\Omega_{\mathrm{opt}}^{2}-\imath \gamma_{\mathrm{opt}} \omega / \omega_{\epsilon}^{2}-\omega^{2} / \omega_{\epsilon}^{2}} \\
\chi^{(2)}(\omega)= & 2 d_{e}\left(1+\frac{C_{0}}{1-\omega^{2} / \omega^{\prime 2}-\imath \Gamma \omega / \omega^{\prime 2}}\right),
\end{aligned}
$$

where $\omega_{\mathrm{THz}}^{2}=0.0729, \quad \omega_{\mathrm{opt}}^{2}=7396, \quad \Omega_{\mathrm{THz}}^{2}=0.0361$, $\Omega_{\mathrm{opt}}^{2}=1225, \quad \gamma_{\mathrm{THz}}=0.0039 \omega_{\epsilon}, \quad \gamma_{\mathrm{opt}}=0.1 \omega_{\epsilon}, \quad \omega_{\epsilon}^{2}=$ $1.78 \times 10^{14}, \quad d_{e}=73 \mathrm{pm} / \mathrm{V}, \quad C_{0}=-0.07, \quad \omega^{\prime}=$ $2 \pi 5.3 \mathrm{THz}$, and $\Gamma=0.017 \omega^{\prime}$.

After solving for the mixed field, a Gabor transform is applied. The Gabor transform produces a two-dimensional function of time and frequency that is equivalent to the output of the BMX-FROG. The results of this numerical model for various parameters are given in the following section.

\section{NUMERICAL RESULTS AND DISCUSSION}

To initially illustrate the capabilities of this technique, the mixing of the self-fields from a short bunch with two different types of probes is examined. The results for a chirped versus a transform limited 500 fs probe are given in Fig. 4. These were produced by mixing the self-fields of a $15 \mathrm{fs}$ bunch with the probe within a $50 \mu \mathrm{m}$ thick ZnTe crystal. The gate pulse for the X-FROG is taken to be a transform limited $100 \mathrm{fs}$ pulse. For the case of the chirped probe, a laser bandwidth of $20 \mathrm{THz}$ was assumed while the transform limited probe has a bandwidth of $\sim 1 \mathrm{THz}$. All quoted values are given as their full width at half-maximum value.

Comparing the probes, it is seen that they are centered at $\sim 0.5 \mathrm{ps}$ and show significant bandwidth broadening. At times trailing the main feature, both have a modulated "tail" that signifies the distortion of the self-fields' profile. This is a direct result of the crystal's resonance. Frequency components above and below the resonance move at different phase velocities leading to a distortion of the self-fields' pulse profile. The primary difference between the two is the linear slope that exists on the chirped pulse which is consistent with the linear chirp that defines the probe.

The reason for such similarities, despite the difference in overall bandwidth, is that the instantaneous bandwidth at any given time is equal to that of the corresponding transform limited pulse, $\sim 1 \mathrm{THz}$. As long as the signal of interest is short compared to the probe, then the bandwidth of the resulting mixed field will be approximately that of the self-fields, regardless of the type of probe used. Put another way, there is no advantage, theoretically, in using one type of probe versus another. However, experimentally, there is an advantage in using a chirped versus a transform limited probe. This is due to how each type of probe is formed. The chirped pulse is produced by taking an ultrashort pulse and putting it through a grating based optical stretcher, where the pulse length is controlled by changing the grating separation. The transform limited probe pulse is produced by passing an ultrashort pulse through a gratingmask system. Here the pulse length is controlled by the mask, which removes frequency components to reduce the overall bandwidth and thus pulse length. The problem with this technique is that the masking process also removes energy from the pulse. Therefore, the peak intensity is less for the transform limited pulse compared to the chirped pulse of the same length. This is detrimental since the mixed field signal is proportional to the probe's field strength.

Having established the effects of a chirp probe on the diagnostic, the output of this technique for various bunch lengths is examined. Figure 5 shows the numerical results for bunch lengths of 5, 15, 50, 100, and 200 fs. Based on these results it has been found that for bunches greater than
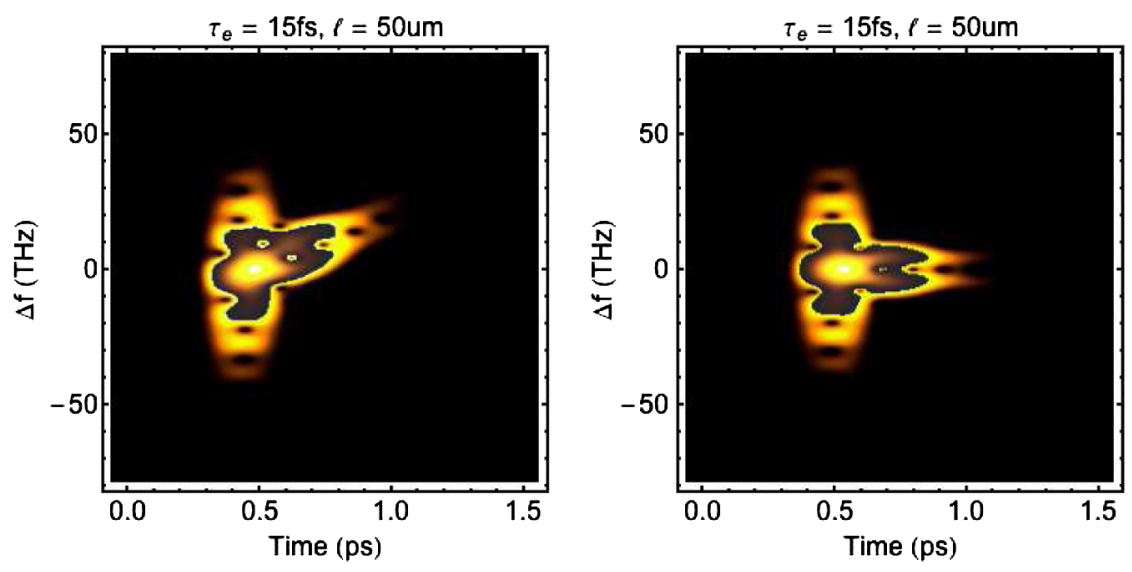

FIG. 4. Comparison between using a chirped (left) and transform limited (right) $500 \mathrm{fs}$ probe pulse to measure a $15 \mathrm{fs}$ bunch through a $50 \mu \mathrm{m}$ crystal. Their total bandwidths are 20 and $\sim 1 \mathrm{THz}$, respectively. Each image is shown using a 100 times filter to emphasize frequency mixing. The unfiltered image is inserted by overlaying it on the saturated portion of the filtered image (central region). 

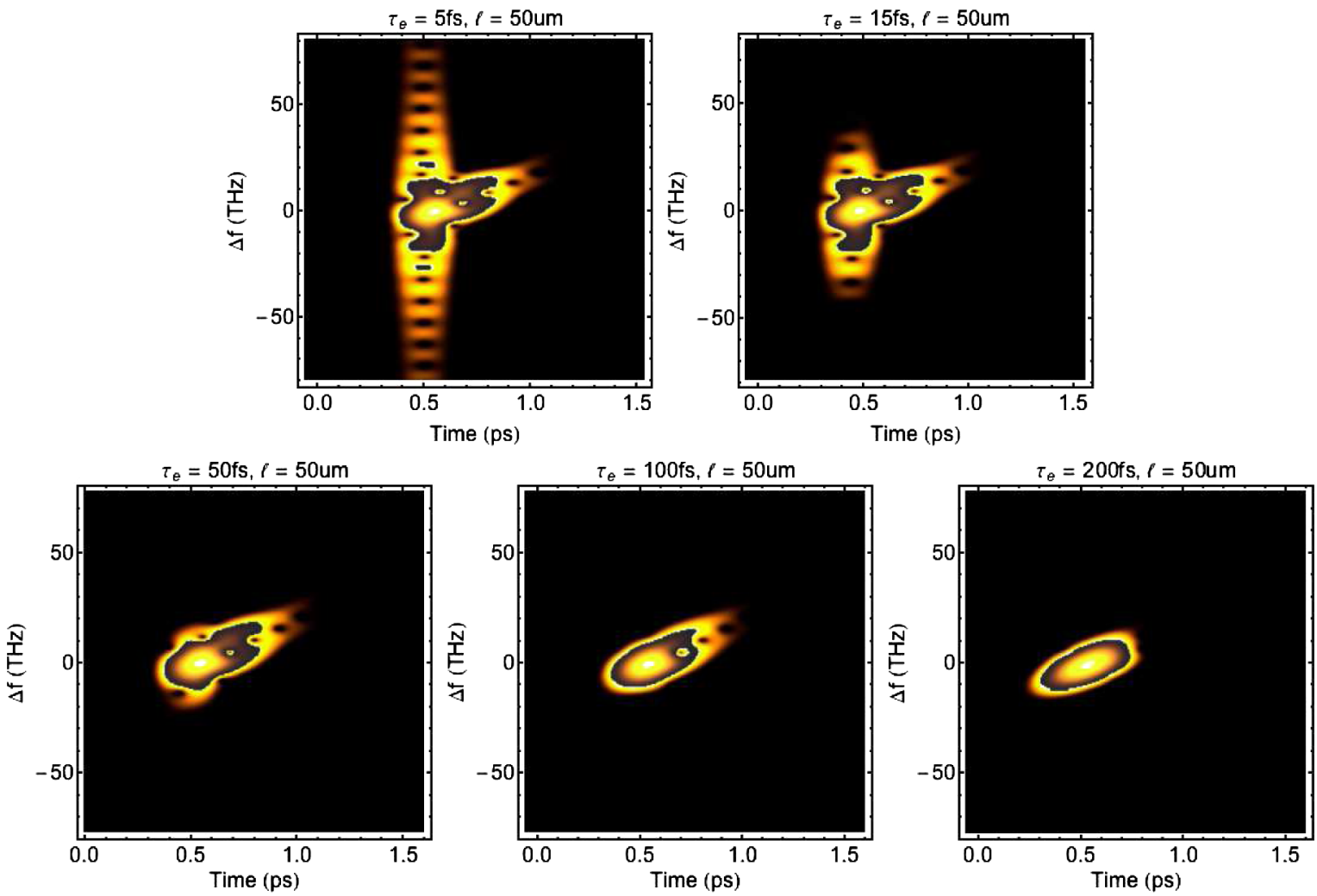

FIG. 5. Simulation results for various bunch lengths measured using a $50 \mu \mathrm{m}$ crystal with a chirped probe. Each image is plotted using its own normalized false color scale.
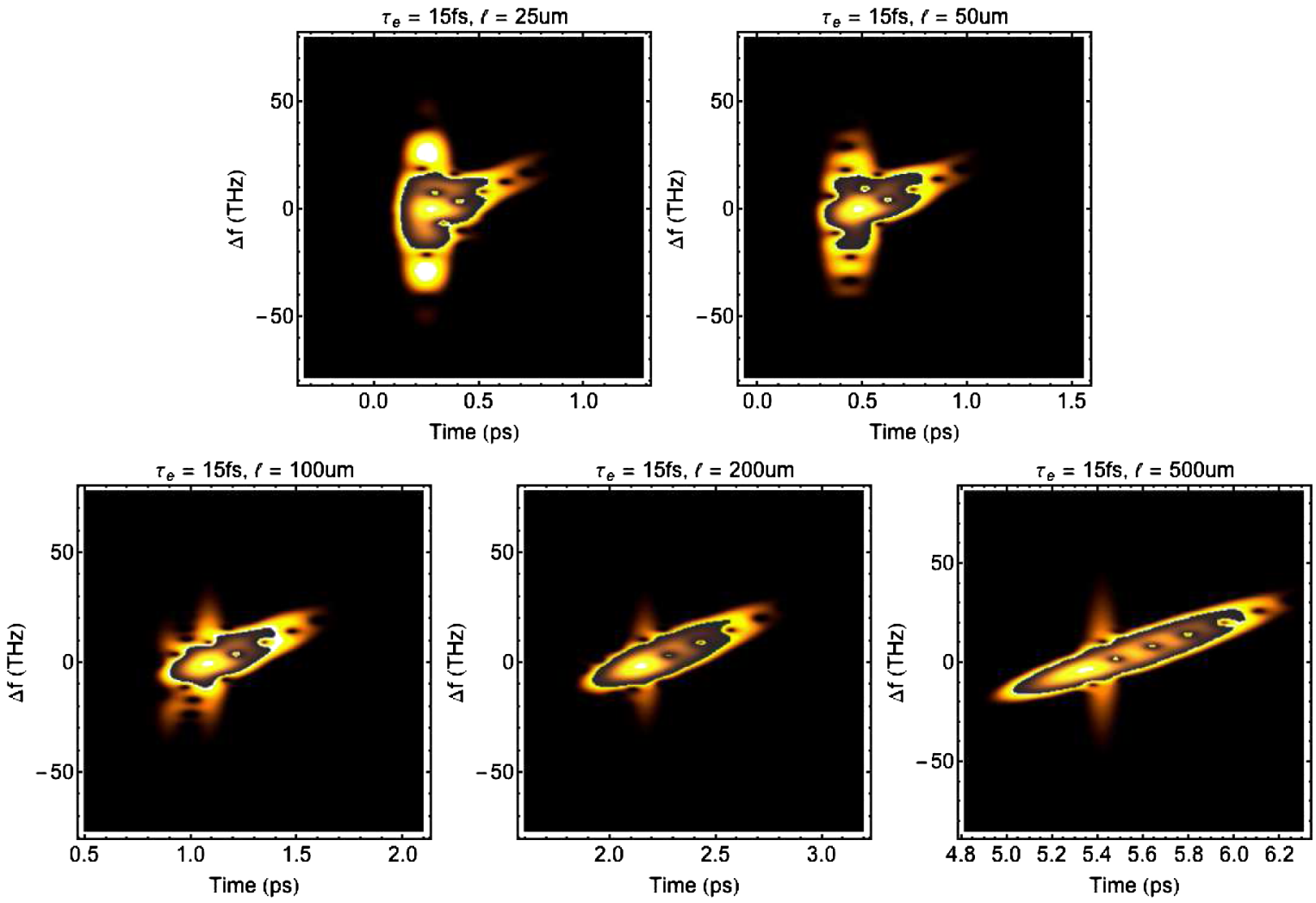

FIG. 6. Simulation results showing the effect of various crystal length on the signal from a $15 \mathrm{fs}$ bunch. 
$\sim 100 \mathrm{fs}$ the temporal profile is faithfully reproduced by integrating the resulting signal over frequency. This is consistent with other techniques [2,3,8]. As the bunch length becomes shorter, the frequency components that define its shape exist on both the low and high frequency sides of the transverse phonon resonance. This leads to a distortion of the self-field's profile as the high frequency components move at a different phase velocity than the low frequency components. However, these higher frequency components can be distinguished by the fact that when they mix with the chirped probe they produce frequency components that extend beyond the bandwidth of the probe pulse. By increasing the sensitivity by a factor of 100 and allowing the low frequency components to saturate the mixing can easily be seen. From these images, it has been found that to first order the bunch length can be extracted by taking the bandwidth of the mixed field and calculating the resulting transform limited pulse. A more faithful bunch profile requires a phase retrieval of the $\mathrm{X}$-FROG image and an inversion of the mixing formalism. This process will be discussed later.

In addition to investigating the output of various bunch lengths, a study of the effects of different crystal thicknesses has been performed. The results for 25, 50, 100, 200, and $500 \mu \mathrm{m}$ thick crystals are shown in Fig. 6. With respect to the low frequency components, it can be seen that they spread out in time as the crystal thickness is increased. This effect is well documented in the literature especially in Ref. [15]. For the high frequency components, it is observed that there are two regions in time where the mixed field is being generated. As the thickness is increased they separate. This is an effect of the dephasing that occurs between the high frequency components of the self-fields and the optical probe. However, the bandwidth is maintained allowing for first order retrieval of the bunch length even when using thick crystals.

While the results given above provide unique insight in the measurement of ultrashort electron beams, it is important to know the consequences of the approximations made in generating Eq. (12) on these results. To examine this, a comparison between the numerical calculation and integration of the exact Maxwell equations is made in the following section.

\section{COMPARISON TO SIMULATIONS}

To self-consistently model the interaction between the self-fields associated with the electron bunch and a dielectric the code TURBOWAVE was used [39]. This was made possible through the recent integration of a nonlinear optics module within the code [35]. Generally, the approach taken was to model the entire interaction within the time domain by associating a set of anharmonic oscillators with each grid cell. Each oscillator contributes to a
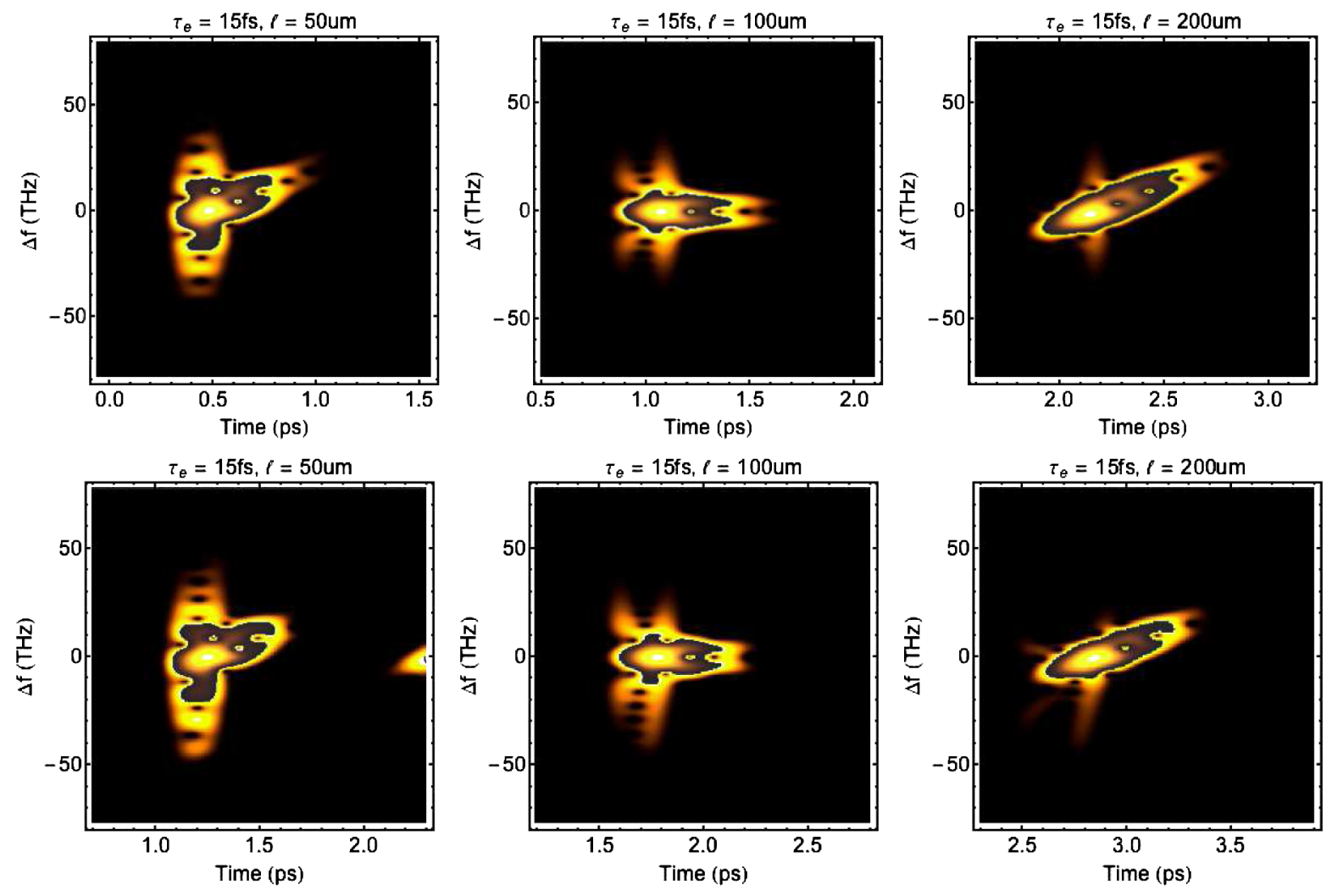

FIG. 7. Comparison between numerical results (top) and results from TURBOWAVE (bottom). 


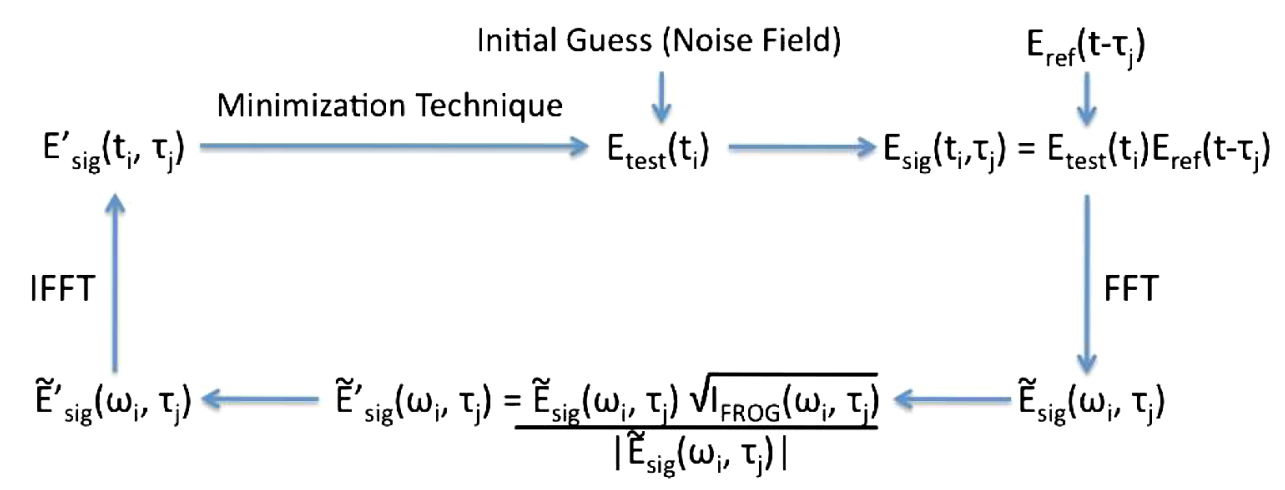

FIG. 8. Schematic of X-FROG retrieval algorithm. $I_{\text {FROG }}$ is the experimental FROG image from which the field is being extracted.

polarization current that acts as an additional source term in the usual Maxwell field solver. The Maxwell solver couples back to the oscillators through the electric field, which appears as a source term in the anharmonic oscillator equation. The standard relationships given in Ref. [30] were used to connect the dispersion relations and second order susceptibility in the frequency domain discussed above to the time domain. The simulation box was set up such that all frequencies up to the electronic resonances were resolved. To ensure a valid comparison, a $500 \mathrm{fs}$ optical probe centered at $800 \mathrm{~nm}$ was used. Also, a 15 fs electron beam with an energy of $250 \mathrm{MeV}$ was used to ensure that the beam was more than sufficiently relativistic. To generate the X-FROG images, the same Gabor transform developed in the numerical calculations was used. A comparison between the results of the numerical calculations and TURBOWAVE are given in Fig. 7 for various crystal thicknesses. An unchirped probe was used in the $100 \mu \mathrm{m}$ case as a double check of the statement made above.

It is apparent after examining the results from the numerical solution with those from TURBOWAVE that they are in good agreement. Upon close inspection there are a couple of minor differences. The first is the positive slope that appears on the frequencies extending beyond the resonance. The lack of this characteristic in the numerical results is a direct consequence of group velocity dispersion (GVD) not being included. GVD is no longer accounted for in the numerical model when the first order expansion of $k(\omega)$ about $\omega_{0}$ was taken. However, solving Eq. (7) directly would correct for this. The second difference is the additional bandwidth observed in the TURBOWAVE results. This is due to the fact that the assumption $\omega \approx \omega_{0}$ begins to break down for frequencies well above the resonance. These are ultimately minor effects and do not adversely effect the physics that is the focus of this work.

\section{MIXED FIELD RECONSTRUCTION}

As mentioned previously, a phase retrieval of the $\mathrm{X}$-FROG image is needed in order to extract a more faithful bunch profile. This is required since FROG images are intensity contours and as such phase information is lost. Once the field is extracted from the X-FROG, the nonlinear mixing formalism must be inverted for the original field profile of the electron beam to be reconstructed. With a sufficient knowledge of the materials dispersion relationships, which are well documented for most common EO materials, this process is relatively straightforward compared to the phase retrieval problem [40]. Equation (12) is simply inverted and the pulse profile is extracted. In order to extract the mixed field, we have developed a phase retrieval algorithm based on the discussions in Trebino [34]. A schematic illustrating the basic algorithm is given in Fig. 8.

The algorithm begins with an initial guess for the retrieved field. This is typically a noise field. The test field is then cross correlated with a well-known reference field, namely the gate pulse. A fast Fourier transform (FFT) is then applied and is modified by the X-FROG image. The resulting field is then transformed back into the time space by an inverse FFT. The final step is to transform the signal field that depends on $t$ and $\tau$ into a field that depends only on $t$. This new field becomes the test field for the next iteration of the phase retrieval algorithm.

We rely on two different techniques to make this transformation. The first is the basis of the "vanilla" FROG algorithm [34]. In this case the signal field is simply integrated over all $\tau$. However, this technique will eventually stagnate. In order to allow the algorithm to reach a more accurate reproduction, a nonlinear conjugate gradient method is used [41]. The code then actively switches between these two techniques as the current one begins to stagnate. This is done until a predefined error or number of iterations is reached. This technique has allowed us to reliably perform phase retrievals with errors of $<10^{-9}$ $[42,43]$. An example of a retrieved field using this technique is shown in Fig. 9. The case used is of the mixed field produced by a $15 \mathrm{fs}$ bunch passing through a $50 \mu \mathrm{m}$ piece of $\mathrm{ZnTe}$. As before, a transform limited probe is used.

At this point, we have the amplitude and phase information for each frequency component of the mixed field as it exits the ZnTe crystal. This information is then used to 

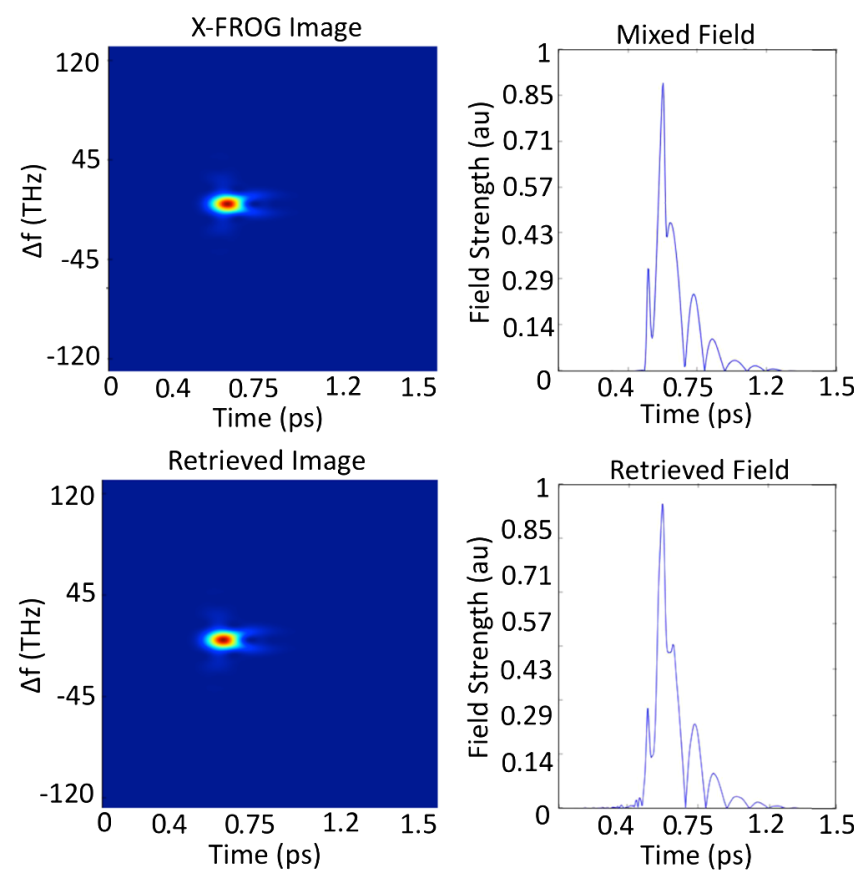

FIG. 9. Comparison between X-FROG (top) and results from phase retrieval algorithm (bottom) for $15 \mathrm{fs}$ electron beam in $50 \mu \mathrm{m}$ of $\mathrm{ZnTe}$. Error was $8 \times 10^{-9}$ for this example.

retrieve the original bunch profile. This is done by inverting Eq. (12) and plugging the retrieved mixed field into it. An example of a retrieved bunch profile is given in Fig. 10. Here the retrieved mixed field shown in Fig. 9 was used. The original $15 \mathrm{fs}$ bunch profile is included as a reference. Also, both profiles are normalized to aid in direct comparison.

The retrieved bunch profile has a FWHM of $17 \mathrm{fs}$. This corresponds to an error of $15 \%$ produced by the retrieval

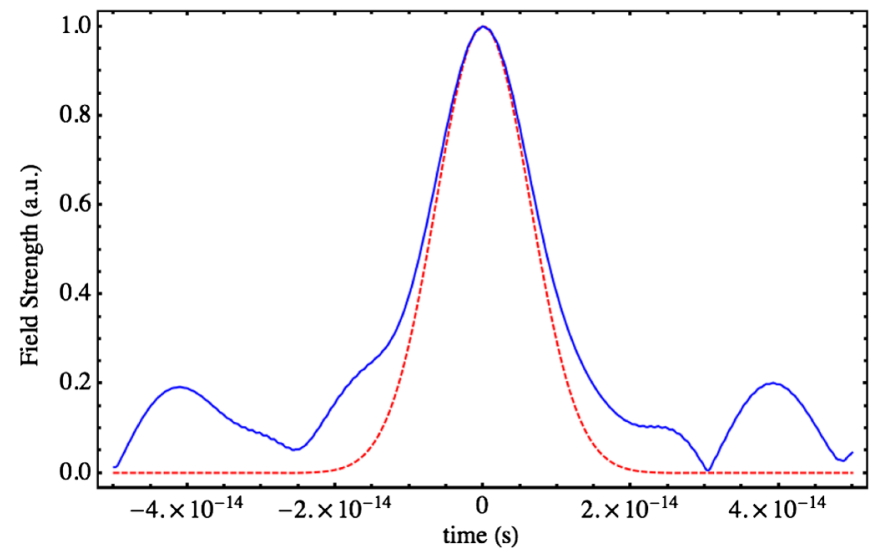

FIG. 10. Bunch profile generated using the results from the XFROG retrieval algorithm and inverting Eq. (12). The original $15 \mathrm{fs}$ bunch profile is shown in red and the retrieved pulse is given in blue. Both curves are normalized for direct comparison. The modulations at the wings of the retrieved pulse are due to the resonance generated in the second order susceptibility. process. This error is a function of the resonance behavior exhibited by the second order susceptibility. This effect is also the cause of the modulated wings on the edges of the retrieved bunch profile. For shorter bunches, where the frequency contributions of this region are lessened, this effect should be diminished; however, other issues due to the large bandwidth of the mixed field may come into play.

\section{CONCLUSION}

Using a three-wave mixing formalism, it can be shown that significant frequency shifts are introduced onto the optical probe of a EO detection scheme for ultrashort beams ( $\lesssim 100 \mathrm{fs}$ ). The BMX-FROG is a device that detects this frequency mixing while simultaneously time resolving the optical probe. Utilizing this enhanced information, the original bunch profile can thus be fully reconstructed.

Numerical calculations based on the mixing formalism were made and are in good agreement with fully explicit nonlinear optics simulations. It was then shown that the bunch profile for a test 15 fs beam can be reconstructed to within an accuracy of $15 \%$. Given a reliable source of ultrashort relativistic electrons, an experimental verification of this technique could be undertaken. In the course, this could prove to be a robust noninvasive beam characterization technique.

\section{ACKNOWLEDGMENTS}

This work was supported by the Naval Research Laboratory Base Program and the Department of Energy. We acknowledge discussions with B. Hafizi and E. Van Keuren.

[1] O. Lundh, J. Lim, C. Rechatin, L. Ammoura, A. BenIsmaill, X. Davoine, G. Gallot, J.-P. Goddet, E. Lefebvre, V. Malka et al., Nature Phys. 7, 219 (2011).

[2] X. Yan, A. M. MacLeod, W.A. Gillespie, G. M. H. Knippels, D. Oepts, A.F.G. van der Meer, and W. Seidel, Phys. Rev. Lett. 85, 3404 (2000).

[3] I. Wilke, A. M. MacLeod, W. A. Gillespie, G. Berden, G. M. H. Knippels, and A. F. G. van der Meer, Phys. Rev. Lett. 88, 124801 (2002).

[4] A. Azima, S. Düsterer, P. Radcliffe, H. Redlin, N. Stojanovic, W. Li, H. Schlarb, J. Feldhaus, D. Cubaynes, M. Meyer et al., Appl. Phys. Lett. 94, 144102 (2009).

[5] A. L. Cavalieri, D. M. Fritz, S. H. Lee, P. H. Bucksbaum, D. A. Reis, J. Rudati, D. M. Mills, P. H. Fuoss, G. B. Stephenson, C. C. Kao et al., Phys. Rev. Lett. 94, 114801 (2005).

[6] G. Berden, S. P. Jamison, A. M. MacLeod, W. A. Gillespie, B. Redlich, and A. F. G. van der Meer, Phys. Rev. Lett. 93, 114802 (2004).

[7] G. Berden, A. F. G. van der Meer, S. P. Jamison, B. Steffen, E.-A. Knabbe, B. Schmidt, P. Schmüser, A. M. MacLeod, P. J. Phillips, and W. A. Gillespie, in Proceedings of the 
10th European Particle Accelerator Conference, Edinburgh, Scotland, 2006 (EPS-AG, Edinburgh, Scotland, 2006), pp. 1055-1057.

[8] S. P. Jamison, G. Berden, A. M. MacLeod, D. A. Jaroszynski, B. Redlich, A.F. G. van der Meer, and W. A. Gillespie, Nucl. Instrum. Methods Phys. Res., Sect. A 557, 305 (2006).

[9] X. Yang, T. Tsang, T. Rao, J. B. Murphy, Y. Shen, and X. J. Wang, Appl. Phys. Lett. 95, 231106 (2009).

[10] G. Berden, W. A. Gillespie, S. P. Jamison, E.-A. Knabbe, A. M. MacLeod, A. F. G. van der Meer, P. J. Phillips, H. Schlarb, B. Schmidt, P. Schmüser et al., Phys. Rev. Lett. 99, 164801 (2007).

[11] B. Steffen, V. Arsov, G. Berden, W. A. Gillespie, S. P. Jamison, A. M. MacLeod, A.F. G. van der Meer, P. J. Phillips, H. Schlarb, B. Schmidt et al., Phys. Rev. ST Accel. Beams 12, 032802 (2009).

[12] J. van Tilborg, C. B. Schroeder, C. V. Filip, C. Tóth, C. G. R. Geddes, G. Fubiani, R. Huber, R. A. Kaindl, E. Esarey, and W. P. Leemans, Phys. Rev. Lett. 96, 014801 (2006).

[13] A. D. Debus, M. Bussmann, U. Schramm, R. Sauerbrey, C. D. Murphy, Z. Major, R. Hörlein, L. Veisz, K. Schmid, J. Schreiber et al., Phys. Rev. Lett. 104, 084802 (2010).

[14] J.D. Jackson, Classical Electrodynamics (Wiley, New York, 1999), 3rd ed.

[15] S. Casalbuoni, H. Schlarb, B. Schmidt, P. Schmüser, B. Steffen, and A. Winter, Phys. Rev. ST Accel. Beams 11, 072802 (2008).

[16] B. Steffen, E.-A. Knabbe, B. Schmidt, P. Schmüser, and A. Winter, in Proceedings of the 27th International Free Electron Laser Conference (SLAC, Palo Alto, CA, 2005).

[17] M. Brunken, H. Genz, P. Gottlicher, C. Hessler, M. Hüning, H. Loos, A. Richter, H. Schlarb, P. Schmüser, S. Simrock et al., TESLA Report No. 11 (2003), pp. 1-24.

[18] N.W. Ashcroft and N.D. Mermin, Solid State Physics (Holt, Rinehart and Winston, New York, 1976).

[19] C. Kittel, Introduction to Solid State Physics (Wiley, New York, 1996), 7th ed.

[20] More exotic electro-optic materials with higher electrooptic coefficients do exist, however they have multiple resonances and their dispersion relations are extremely complex [21].

[21] Y. Takahashi, H. Adachi, T. Taniuchi, M. Takagi, Y. Hosokawa, S. Onzuka, S. Brahadeeswaran, M. Yoshimura, Y. Mori, H. Masuhara et al., J. Photochem. Photobiol., A 183, 247 (2006).

[22] G. Gallot and D. Grischkowsky, J. Opt. Soc. Am. B 16, 1204 (1999).

[23] Recent experimental work by Jamison et al. has shown the frequency mixing that occurs when the self-fields of an $50 \mathrm{MeV} 650$ fs FWHM electron beam mixes with a quasimonochromatic Ti:sapphire laser pulse [28].

[24] M. H. Helle, D. F. Gordon, D. Kaganovich, E. V. Keuren, and A. Ting, AIP Conf. Proc. 1299, 561 (2010).
[25] S.P. Jamison, A. M. MacLeod, G. Berden, D. A. Jaroszynski, and W. A. Gillespie, Opt. Lett. 31, 1753 (2006).

[26] F. J. P. Wijnen, G. Berden, and R. T. Jongma, Opt. Express 18, 26517 (2010).

[27] Z. Chen, Y. Gao, and M. F. DeCamp, Appl. Phys. Lett. 99, 011106 (2011).

[28] S. P. Jamison, G. Berden, P. J. Phillips, W. A. Gillespie, and A. M. MacLeod, Appl. Phys. Lett. 96, 231114 (2010).

[29] Y. R. Shen, The Principles of Nonlinear Optics (J. Wiley, New York, 1984).

[30] R. W. Boyd, Nonlinear Optics (Academic Press, Amsterdam, 2008), 3rd ed.

[31] W. Koechner, Solid-state Laser Engineering (Springer, New York, 2006), Vol. 1, 6th ed.

[32] P. R. Bolton, J. E. Clendenin, D. H. Dowell, P. Krejcik, and J. Rifkin, Nucl. Instrum. Methods Phys. Res., Sect. A 507, 220 (2003).

[33] P. R. Bolton, Int. J. Mod. Phys. B 21, 527 (2007).

[34] R. Trebino, Frequency-Resolved Optical Gating: The Measurement of Ultrashort Laser Pulses (Kluwer Academic Publishers, Norwell, MA, 2000).

[35] D. F. Gordon, M. H. Helle, D. Kaganovich, and A. Ting, AIP Conf. Proc. 1299, 67 (2010).

[36] G. Gallot, J. Zhang, R. W. McGowan, T.-I. Jeon, and D. Grischkowsky, Appl. Phys. Lett. 74, 3450 (1999).

[37] Differences between the model and the numerical solution arise in the UV range, however these frequencies are beyond those considered in this work. Also, apparent discrepancies in the extinction coefficient are visually emphasized due to the vertical log scale. The discrepancies become appreciable only for low values that do not contribute.

[38] CRC Handbook of Chemistry and Physics, edited by W. M. Haynes and D. R. Lide (Chapman and Hall/ CRCnetBASE, Boca Raton, FL, 2009), Vol. 90.

[39] D. F. Gordon, IEEE Trans. Plasma Sci. 35, 1486 (2007).

[40] Issues with inversion of the mixing formalism may arise due to the combination of material absorption and the finite dynamic range of experiments. This may need to be addressed at that time.

[41] J. Nocedal and S. Wright, Numerical Optimization (Springer, New York, 1999).

[42] Noise on the signal could prove to be an experimental issue due to the factor of 100 difference between the main feature and frequency mixing wings. However, we believe this could be compensated for by using a holographic notch filter or placing a mask in the imaging spectrometer.

[43] Error measurements are made by calculating the sum of least squares between the X-FROG image and the retrieved image. This error measure differs from that described in Trebino [34], thus care should be taken when making direct comparisons between our technique and others.

[44] H. D. Riccius and R. Turner, J. Phys. Chem. Solids 29, 15 (1968). 